\title{
Pharmacologic cerebral capillary blood flow improvement after deep hypothermic circulatory arrest: An intravital fluorescence microscopy study in pigs
}

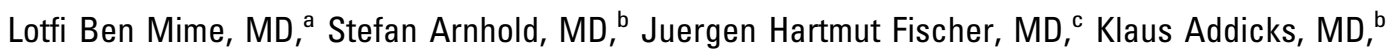

Ernst Rainer de Vivie, MD, ${ }^{a}$ Gerardus Bennink, MD, ${ }^{a}$ and Michael Suedkamp, MD ${ }^{a}$

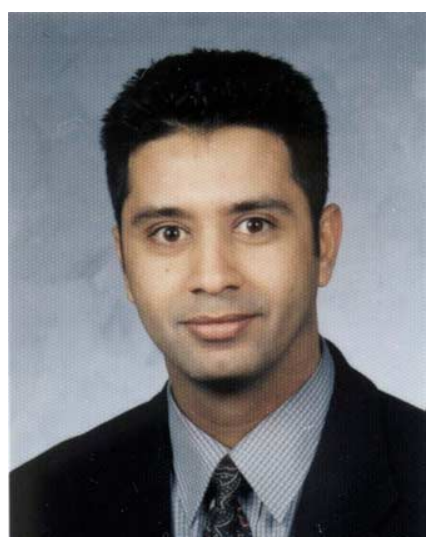

Dr Ben Mime
From the Departments of Cardiothoracic Surgery, ${ }^{\mathrm{a}}$ Anatomy, ${ }^{\mathrm{b}}$ and Experimental Medicine, ${ }^{\mathrm{c}}$ University of Cologne, Cologne, Germany.

Read at the Thirtieth Annual Meeting of The Western Thoracic Surgical Association, Maui, Hawaii, June 23-26, 2004.

Received for publication Nov 23, 2004; revisions accepted Feb 24, 2005; accepted for publication March 21, 2005.

Address for reprints: Lotfi Ben Mime, MD, Department of Cardiothoracic Surgery, University of Cologne, Josef-Stelzmann-Strasse 9, 50924 Cologne, Germany (E-mail: Lofti.ben-mime@uk-koeln.de).

J Thorac Cardiovasc Surg 2005;130:670-6

$0022-5223 / \$ 30.00$

Copyright (C) 2005 by The American Association for Thoracic Surgery

doi:10.1016/j.jtcvs.2005.03.035
Background: Despite meticulous investigation of bypass techniques for deep hypothermic circulatory arrest, unfavorable long-term neurologic deficits have been well documented. Our aim was to improve brain perfusion by reducing platelet plugging with a glycoprotein IIb/IIIa inhibitor (eptifibatide) in an experimental model of deep hypothermic circulatory arrest-reperfusion in pigs.

Methods: Two groups of 12 piglets each (eptifibatide group [eptifibatide + unfractionated heparin] vs UFH group [only unfractionated heparin]) underwent 10 minutes of normothermic bypass, 40 minutes of cooling during cardiopulmonary bypass (hematocrit, $30 \%$; cardiopulmonary bypass flow, $100 \mathrm{~mL} \cdot \mathrm{kg}^{-1} \cdot \mathrm{min}^{-1}$ ), 60 minutes of circulatory arrest at $15^{\circ} \mathrm{C}$, and a 40 -minute rewarming period. Intravital fluorescence microscopy of pial vessels at set intervals was performed.

Results: During the cooling period, there was a tendency toward reduced functional capillary density values without statistical significance in both groups. During reperfusion, the eptifibatide group demonstrated a significantly decreased platelet adhesion and aggregation (at 30 minutes of reperfusion: functional capillary density, $104 \% \pm 3 \%$ vs $77 \% \pm 4 \%$ relative to baseline, $P=.02$; red blood cell velocity, 0.65 vs $0.30 \mathrm{~mm} / \mathrm{s}, P<.004)$. A more rapid recovery of tissue oxygenation $(P<$ $.001)$ was documented. Furthermore, a significant microvascular permeability reduction was achieved compared with that seen in the UFH group $(P<.02)$. The use of eptifibatide resulted in fewer ultrastructural changes in hippocampal tissue, which is demonstrated by histologic examination.

Conclusions: Platelet plugging reduction with the glycoprotein IIb/IIIa inhibitor eptifibatide improves cerebral capillary blood flow and reduces cerebral ischemia in the setting of deep hypothermic circulatory arrest. Furthermore, significant endothelial cell injury and perivascular edema reduction can be achieved.

I $\mathrm{n}$ the setting of deep hypothermic circulatory arrest (DHCA)-reperfusion hypoxia, various shear stress rates on cardiopulmonary bypass (CPB) during cooling, circulatory arrest, reperfusion, heat stress, oxidative stress, homotypic and heterotypic blood cell interactions, and activation are just a few of the various processes inducing cellular stress, subsequently damaging function and structures. Brain damage is mainly caused by uneven brain reperfusion related to capillary changes implicated in the no-reflow phenomenon. ${ }^{1,2}$ Hypoxic endothelial cell injury leads to procoagulant response, resulting in intravascular microthrombosis, which is enhanced by neutrophil and platelet plugging. ${ }^{4-8}$ When organ (eg, heart and brain) ischemia caused by DHCA of 10 to 20 minutes' duration is induced, rapid depletion of high-energy phosphates (eg, adenosine triphosphate [ATP]) is seen, along with mild tissue and mitochondrial swelling and loss of glycogen stores. ${ }^{9}$ Cell membranes remain intact, and the cells are still viable. Reperfusion at this time allows 
replacement of high-energy phosphates and reversal of ischemic structural changes. ${ }^{3}$ Cellular injury, however, becomes irreversible with prolonged ischemia. Anaerobic glycolysis ceases, and sarcolemmal and mitochondrial changes occur. Functioning mitochondria are needed for the resynthesis of ATP during reperfusion. Reperfusion at this time can accelerate the destruction of irreversibly injured cells. With more prolonged ischemia, reperfusion might not occur at all. Possible reasons for the inability to reperfuse include capillary compression caused by aggregation of blood cell components, endothelial and parenchymal compression from edema, and swelling of cells (glia and neurons). ${ }^{2}$ Glycoprotein IIb/IIIa receptor blockade, apart from its established effects on maintaining large-vessel patency and enhancing microvascular myocardial perfusion, has been found to improve vascular nitric oxide bioavailability, which is involved in vasomotor regulation. ${ }^{10}$ We hypothesized that a significantly improved cerebral reperfusion after DHCA could be achieved by reducing plug formation, especially at the capillary level, and by preserving endothelial vasomotor function. This could, in turn, reduce the abovementioned ultrastructural brain deterioration.

\section{Methods}

\section{Surgical Procedure}

Twenty-four experiments ( $\mathrm{n}=12$ in each experimental group) were performed on 3- to 4-week-old hybrid pigs (Piétrain Halothan-Gen -/- and German Country Pedigree) with an average body weight of 10 to $15 \mathrm{~kg}$. All animals received humane care in compliance with the "Guide for the Care and Use of Laboratory Animals" published by the National Institutes of Health (NIH publication no. 85-23, revised 1985). After intramuscular premedication with ketamine $(20 \mathrm{mg} / \mathrm{kg}$ ) and xylazine $(4 \mathrm{mg} / \mathrm{kg})$, endotracheal intubation (cuffed 5-mm tube), and a bolus of fentanyl (25 $\mu \mathrm{g} / \mathrm{kg}$ administered intravenously), the piglets were artificially ventilated with a pressure-controlled respirator by an inspiratory oxygen fraction of 50\% before CPB and $100 \%$ after CPB at a rate of between 18 to 22 breaths $/ \mathrm{min}$ to achieve an arterial $\mathrm{PCO}_{2}$ of between 35 and $40 \mathrm{~mm} \mathrm{Hg}$. Anesthesia was maintained with fentanyl $\left(25 \mu \mathrm{g} \cdot \mathrm{kg}^{-1} \cdot \mathrm{h}^{-1}\right)$, midazolam $\left(0.2 \mathrm{mg} \cdot \mathrm{kg}^{-1} \cdot \mathrm{h}^{-1}\right)$, and pancuronium $\left(0.2 \mathrm{mg} \cdot \mathrm{kg}^{-1} \cdot \mathrm{h}^{-1}\right)$ by an infusion pump. A rectal temperature probe was placed. The right femoral artery was cannulated, and the catheter was advanced into the descending aorta for monitoring of blood pressure and blood gases. Blood pressure and body temperature were continuously monitored and recorded every 10 minutes. Blood gases were checked for $\mathrm{PO}_{2}$, $\mathrm{PCO}_{2}, \mathrm{pH}$, potassium, and lactate every 10 minutes on $\mathrm{CPB}$ in 1-mL samples by a blood-gas analyzer (ABL 625, Radiometer Medical A/S, Copenhagen, Denmark). A catheter was placed through the right femoral vein into the inferior vena cava for infusion of drugs and fluorescent dyes. The aorta was exposed for arterial CPB cannulation through a right anterolateral thoracotomy in the third intercostal space. After administration of heparin (300 $\mathrm{IU} / \mathrm{kg}$ ), an arterial cannula (8F; BioMedicus, Eden Prairie, Minn) was advanced through the aorta. A $24 \mathrm{~F}$ venous cannula (Stoeckert, Munich, Germany) was inserted into the right atrium. The heart was not opened during the procedure. Immediately after insertion of the catheter into the inferior vena cava, the eptifibatide group received a bolus of eptifibatide $(180 \mu \mathrm{g} / \mathrm{kg})$ twice at 10 -minute intervals, followed by continuous eptifibatide administration with an infusion pump $\left(20 \mu \mathrm{g} \cdot \mathrm{kg}^{-1} \cdot \mathrm{min}^{-1}\right)$. This infusion was then stopped at the beginning of DHCA. The animals were placed in a prone position, and a left-sided trepanation over the left parietal cortex was performed with an electric drill. The final size of the hole in the skull was $15 \times 25 \mathrm{~mm}$. Bleeding from the bone and periosteum of the fully heparinized animals was controlled with bone wax. After incision of the dura, the pial vessels were visualized.

\section{Intravital Microscopy: Hardware Configuration and Measurements}

The microscopy facility used for intravital microscopy of the brain microcirculation in anesthetized pigs is a combination of a Leica stereo epifluorescence microscope (model MZFL III Fluo Combi; Leica, Heerbrugg, Switzerland) with a 100-W mercury gas discharge lamp equipped with a rapid filter exchanger (including 3 sets of filters) and the Leica Fluo Combi, a 3 Chip CCD camera (Hitachi HV-C20) and a computer workstation. The microscope was placed above the cranial window. The images from the 3 Chip CCD camera were displayed on a high-resolution 15 -inch monitor. The microscopic images were also recorded with an S-VHS video recorder on S-VHS tapes to back up the collected data. The final magnification on the monitor was $400 \times$. The analysis of the recorded images was performed offline. To visualize microvascular (diameter range, 15-180 $\mu \mathrm{m}$ ) perfusion, plasma was labeled with 5\% fluorescein isothiocyanate (FITC)-dextran (molecular weight, 150,000 d; Sigma Aldrich, Munich, Germany) by intravascular injection of $2 \mathrm{~mL}$ of FITC-dextran for baseline recordings and $0.5 \mathrm{~mL}$ of FITC-dextran before each subsequent measurement (intravenous injection before and after $\mathrm{CPB}$ and intra-arterial injection on CPB). FITC fluorescence was excited with a 450- to 490-nm light, and the emitted light (>515 nm; blue filter set, Leica) was transferred from the microscope to a video camera. Thus, the plasma was highlighted, and the red blood cells appeared dark (negative contrast). The window was regularly washed with physiologic saline solution to minimize heating of the cover slip and the underlying tissue. For observation of leukocyteendothelial cell interactions, the circulating leukocytes were labeled by intravenous injection of $2 \mathrm{~mL}$ of Rhodamine $6 \mathrm{G}$ solution (Sigma Aldrich) before each measurement. A green filter set (excitation wavelength, $536-556 \mathrm{~nm}$; emission wavelength, $>590$ $\mathrm{nm}$; Leica) was used to excite Rhodamine fluorescence. Microvascular perfusion and reduced nicotinamide adenine dinucleotide (NADH) fluorescence were recorded every 10 to 15 minutes on CPB. In addition, NADH fluorescence was recorded every 15 minutes during circulatory arrest. The duration of epi-illumination was limited to 1 minute at set intervals to avoid thermal injury of tissue. The epi-illumination of brain tissue was always shut off between video recordings.

\section{Functional Capillary Density}

After focusing below the level of the pial vessels, the cortical capillaries could be visualized because of the FITC fluorescence of blood plasma. Functional capillary density (FCD), defined as the 
TABLE 1. Experimental conditions

\begin{tabular}{|c|c|c|c|c|c|c|c|}
\hline Parameter & Group & Pre-CPB & $10^{\prime} \mathrm{NT}$ & $10^{\prime} \mathrm{cool}$ & End cool & $10^{\prime}$ rewarm & End rewarm \\
\hline \multirow[t]{2}{*}{ Arterial pH } & Eptifibatide group & $7.6 \pm 0.06$ & $7.5 \pm 0.09$ & $7.4 \pm 0.1$ & $7.4 \pm 0.06$ & $7.4 \pm 0.1$ & $7.5 \pm 0.19$ \\
\hline & UFH group & $7.6 \pm 0.08$ & $7.5 \pm 0.09$ & $7.4 \pm 0.1$ & $7.36 \pm 0.05$ & $7.4 \pm 0.1$ & $7.4 \pm 0.13$ \\
\hline \multirow[t]{2}{*}{ Arterial $\mathrm{PCO}_{2}$} & Eptifibatide group & $33.4 \pm 7$ & $45.5 \pm 10.8$ & $51 \pm 10.4$ & $50.4 \pm 7$ & $34.9 \pm 9.1$ & $31.3 \pm 9.2$ \\
\hline & UFH group & $35.1 \pm 7.4$ & $37.6 \pm 6.5$ & $44.5 \pm 11.4$ & $48.6 \pm 9.3$ & $37.4 \pm 7.9$ & $29.7 \pm 9.6$ \\
\hline \multirow[t]{2}{*}{ Arterial $\mathrm{PO}_{2}$} & Eptifibatide group & $426.5 \pm 97.4$ & $207.8 \pm 175.7$ & $489.7 \pm 291.7$ & $530.5 \pm 339.2$ & $286 \pm 206.1$ & $310.1 \pm 187.1$ \\
\hline & UFH group & $426.7 \pm 126.9$ & $355.2 \pm 196.3$ & $518.9 \pm 317.7$ & $342.7 \pm 374.3$ & $338.9 \pm 248.7$ & $298.5 \pm 181.5$ \\
\hline \multirow{2}{*}{$\begin{array}{l}\text { Arterial } \\
\text { hematocrit }\end{array}$} & Eptifibatide group & $29.1 \pm 1.7$ & $29.3 \pm 3$ & $29.3 \pm 1.7$ & $29 \pm 3$ & $29.6 \pm 3.4$ & $28.6 \pm 5$ \\
\hline & UFH group & $26.9 \pm 2$ & $30 \pm 3.6$ & $29.7 \pm 3.2$ & $28.8 \pm 1.6$ & 29.55 & $30.6 \pm 3.7$ \\
\hline \multirow{2}{*}{$\begin{array}{l}\text { Rectal } \\
\text { temperature }\end{array}$} & Eptifibatide group & $37.8 \pm 0.8$ & $35.1 \pm 1.8$ & $26.7 \pm 2.9$ & $15.7 \pm 0.9$ & $29.6 \pm 4.1$ & $36.2 \pm 1.8$ \\
\hline & UFH group & $38 \pm 0.0$ & $33.8 \pm 2.2$ & $19.9 \pm 2.05$ & $15.6 \pm 0.8$ & $30 \pm 2.2$ & $36.5 \pm 1.1$ \\
\hline \multirow{2}{*}{$\begin{array}{l}\text { Mean arterial } \\
\text { pressure }\end{array}$} & Eptifibatide group & $88.6 \pm 12.9$ & $82.3 \pm 18.1$ & $85.3 \pm 27.6$ & $77.5 \pm 14$ & $69.8 \pm 26.9$ & $83.7 \pm 18.5$ \\
\hline & UFH group & $72.8 \pm 16.6$ & $87.6 \pm 23.9$ & $74.6 \pm 18.5$ & $69.6 \pm 16.7$ & $58.3 \pm 16.1$ & $77.2 \pm 21.5$ \\
\hline \multirow[t]{2}{*}{$\begin{array}{l}\text { Arterial } \\
\text { lactate }\end{array}$} & Eptifibatide group & $2 \pm 0.6$ & $4.1 \pm 1.9$ & $5.2 \pm 2.4$ & $5.6 \pm 1.8$ & $8.8 \pm 2.3$ & $10.3 \pm 2.2$ \\
\hline & UFH group & $1.7 \pm 0.5$ & $4.2 \pm 1.4$ & $5 \pm 1.74$ & $5.8 \pm 2.07$ & $10.4 \pm 2$ & $12 \pm 3.84$ \\
\hline \multirow{2}{*}{$\begin{array}{r}\text { Platelet } \\
\text { count }\end{array}$} & Eptifibatide group & $(419 \pm 30)^{*} 10^{3}$ & & & & & $(279 \pm 32)^{*} 10^{3}$ \\
\hline & UFH group & $(301 \pm 22)^{*} 10^{3}$ & & & & & $(104 \pm 25)^{*} 10^{3}$ \\
\hline
\end{tabular}

Values for arterial $\mathrm{pH}, \mathrm{PCO}_{2}$, and $\mathrm{PO}_{2}$ are corrected to rectal temperature. Pre-CPB, Baseline values before cardiopulmonary bypass; $10^{\prime}$ NT, after 10 minutes of normothermic bypass; 10' cool, after 10 minutes of cooling; End cool, end of cooling; 10' rewarm, after 10 minutes of rewarming; End rewarm, end of rewarming; Platelet count, pre-CPB compared with the end of rewarming, significantly higher platelet count reduction in UFH group compared with the study group $(P<.05)$.

total length of red blood cell-perfused capillaries per observation area (in centimeters), was determined as previously described by Duebener and colleagues. ${ }^{11}$

\section{Tissue Oxygenation}

The natural intracellular fluorophore NADH, which accumulates during ischemia, was used as a marker for tissue oxygenation. NADH fluorescence was excited with a 340- to 380-nm light (UV-Filter set: emission wavelength, $>420 \mathrm{~nm}$; Leica). During these measurements, the remote control of the video camera for brightness and contrast was disabled. The optical densities (black $=0$, white $=255$ ) of the recorded still images were determined by densitometry.

\section{Red Blood Cell Velocity}

Microscopic images of the moving red blood cells were collected with the high-speed 3 Chip CCD camera. A sequence at the median plane of each vessel was recorded for offline analysis of red blood cell velocity (RBCV) with the Cap-Image line-shiftdiagram method (Cap-Image; Ingenieurbüro Zeintl, Heidelberg, Germany).

\section{Microvascular Permeability}

The extravasation of FITC-dextran-labeled plasma through disrupted endothelial cell lining resulted in the formation of confluent dye clouds, which surround the microvessels. The perivascular optical density changes that occurred during the experiment were determined by densitometry.

\section{Bypass Management}

After baseline recordings of the cerebral microcirculation, CPB was initiated. A roller pump (Stoeckert) was used to generate a nonpulsatile pump flow of $100 \mathrm{~mL} / \mathrm{kg}$ body weight in all experiments. The gas flow was adjusted to achieve an arterial $\mathrm{PCO}_{2}$ of 40 to $45 \mathrm{~mm} \mathrm{Hg}$. The CPB circuit in all experiments consisted of a 1000-mL filtered hard-shell venous reservoir (Cobe VPCML Plus; COBE Cardiovascular, Inc, Arvada, Colo), a membrane oxygenator, a $40-\mu \mathrm{m}$ arterial filter, and $1 / 4$-inch tubing. Venous drainage was by gravity. No cardiotomy suction was used. The venous line was left open during circulatory arrest. A sterile circuit was used in each experiment. The CPB circuit was primed with $800 \mathrm{~mL}$ of blood. The blood used for priming of the $\mathrm{CPB}$ circuit to achieve a hematocrit level of $30 \%$ on CPB was drawn on the morning of the experiment from an adult donor pig. Before the start of $\mathrm{CPB}$ and during reperfusion, methylprednisolone (25 mg/kg), cephazolin $(25 \mathrm{mg} / \mathrm{kg})$, $10 \mathrm{~mL}$ of sodium bicarbonate $7.4 \%$, and furosemide $(0.25$ $\mathrm{mg} / \mathrm{kg}$ ) were added to the prime. After 10 minutes of normothermic bypass, piglets underwent 40 minutes of cooling to a rectal temperature of $15^{\circ} \mathrm{C}$. After 60 minutes of DHCA, animals were rewarmed on $\mathrm{CPB}$ to $37^{\circ} \mathrm{C}$. After weaning from $\mathrm{CPB}$, the animals were observed for 30 minutes. The room temperature in the animal operating room was thermostatically controlled (range, $15^{\circ} \mathrm{C}$ to $30^{\circ} \mathrm{C}$ ) to aid cooling and rewarming of animals. No topical cooling was applied.

Experimental conditions are shown in Table 1. 


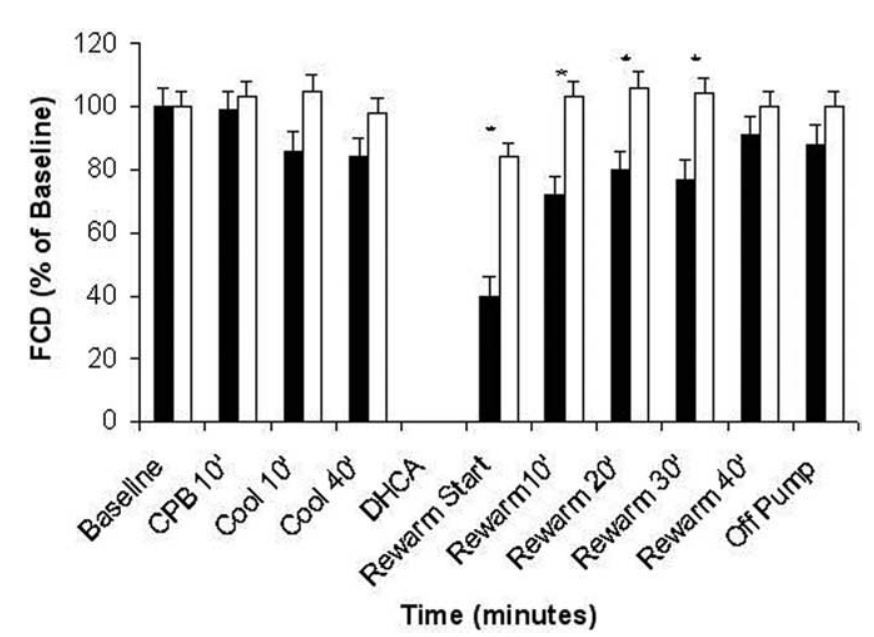

Figure 1. FCD values were significantly lower in the UFH group during early reperfusion (reperfusion start and 30 minutes of reperfusion: $P<.007$ and $>P=.02$, respectively). Filled columns, UFH group; open columns, eptifibatide group. ${ }^{*} P<.05$.

\section{Histopathologic Analysis}

At the end of the experiment, the animals underwent bilateral carotid perfusion with $4 \%$ paraformaldehyde in $0.1 \mathrm{~mol} / \mathrm{L}$ phosphate buffer. The brain was then removed and dissected in smaller tissue samples. The hippocampus was isolated, and a standard postfixation was then performed. Ultrathin sections were cut with an ultramicrotome (Reichert), and they were examined with a Zeiss EM 902 electron microscope (Carl Zeiss AG, Gottingen, Germany).

\section{Statistical Analysis}

Continuous variables are expressed as means \pm standard deviation. Variables were tested for normality by the KolmogorovSmirnov goodness-of-fit test, and no significant skewness was detected. Repeated-measures analysis of variance was performed to evaluate changes over time and to compare rates of change between the groups. For time-point comparisons within an experimental group, paired $t$ tests were used. One-way factorial analysis of variance with the post-hoc Bonferroni method was used to evaluate group differences at fixed time points. Data analysis was conducted with the SPSS software package (release 11.0; SPSS, Inc, Chicago, Ill).

\section{Results}

\section{Functional Capillary Density}

There was a slight decrease of FCD relative to baseline values during cooling in both groups. At the end of cooling, the FCD was $98 \% \pm 2 \%$ in the eptifibatide group and $84 \%$ $\pm 3 \%$ in the unfractionated heparin (UFH) group $(P=.17)$. During early reperfusion, there were significantly higher FCD values in the eptifibatide group relative to the UFH group (reperfusion start: $84 \% \pm 3 \%$ [eptifibatide] vs $40 \% \pm$ $4 \%$ [UFH],$P<.007 ; 30$ minutes of reperfusion: $104 \% \pm$

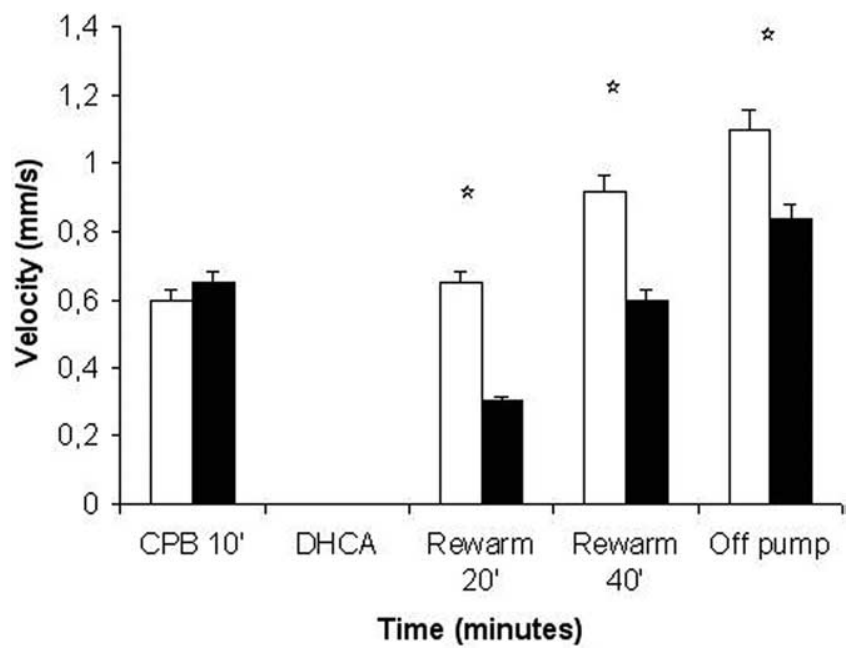

Figure 2. RBCV recovery during reperfusion was more rapid in the study group. At the end of rewarming, there was still a significant difference between both groups. Furthermore, the RBCV value in the UFH group at $\mathbf{4 0}$ minutes of reperfusion was significantly lower than the pre-CPB value $(P<.05)$. Filled columns, UFH group; open columns, eptifibatide group. ${ }^{*} P<.05$.

$3 \%$ vs $77 \% \pm 4 \%, P=.02$ ). At the end of reperfusion and 30 minutes after weaning from CPB, the FCD was not significantly different from baseline values in either group (Figure 1).

\section{Capillary Red Blood Cell Velocity}

A significant cortical capillary RBCV reduction was observed after 10 minutes of CPB in both groups. During reperfusion, there was a rapid and sustained recovery of capillary RBCV in the eptifibatide group. The UFH group showed a delayed RBCV recovery (20 minutes of reperfusion: velocity of $0.65 \mathrm{vs} 0.30 \mathrm{~mm} / \mathrm{s}$ in the eptifibatide and UFH groups, respectively; $P<.004$ ), and this did not return to the normal value or equal value to the eptifibatide group (Figure 2).

\section{Tissue Oxygenation}

The NADH autofluorescence of the parietal cortex slightly decreased in the eptifibatide group at the end of cooling, indicating better cerebral tissue oxygenation. At the same time point, the UFH group showed no significant changes relative to baseline. During early DHCA (at 10 minutes of DHCA), the NADH autofluorescence reached $96 \% \pm 2 \%$ and $124 \% \pm 3 \%$ in the eptifibatide and UFH groups, respectively $(P=.05)$, indicating significantly poorer tissue oxygenation in the UFH group. This more severe deoxygenation in the UFH group was sustained during the entire DHCA period and the early reperfusion period (10 minutes of reperfusion: $98 \% \pm 2 \%$ [eptifibatide] vs $128 \% \pm 3 \%$ 


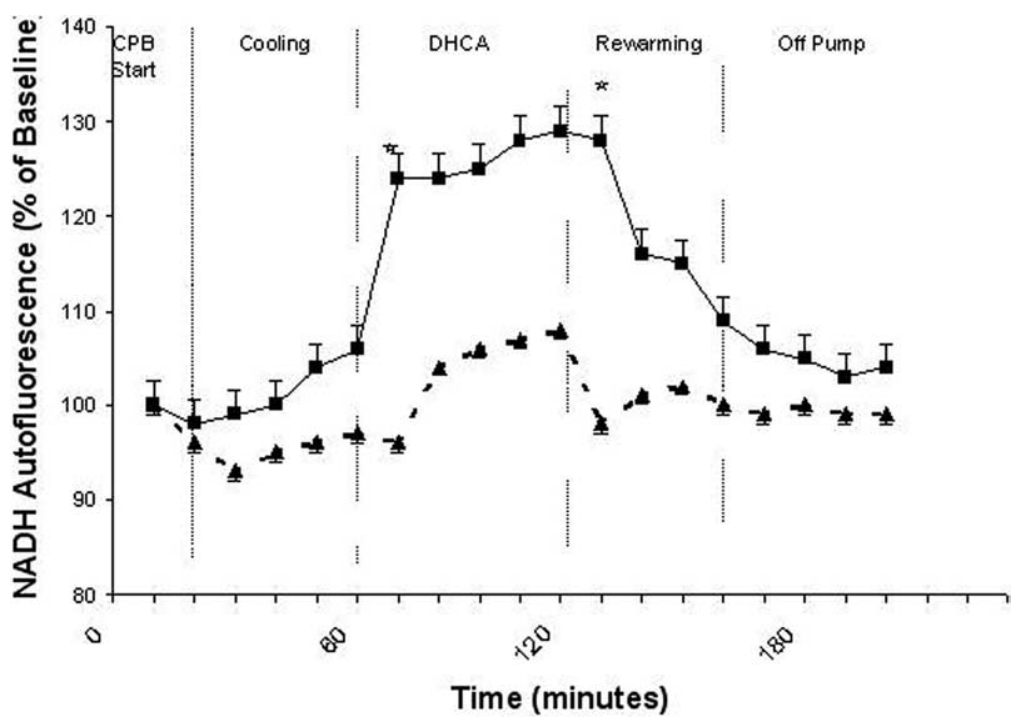

Figure 3. NADH autofluorescence. Tissue oxygenation was significantly lower (higher NADH autofluorescence) in the UFH group during DHCA and early rewarming (10 minutes of DHCA, $P=.05$; 10 minutes of rewarming, $P<.04)$. Triangles, Eptifibatide group; squares, UFH group. ${ }^{*} P<.05$.

[UFH], $P<.04)$. Furthermore, the recovery time to reach baseline values was significantly shorter in the eptifibatide group ( 5 vs 40 minutes, $P<.001$ ). After 10 minutes of reperfusion, there was a complete metabolic recovery in the eptifibatide group, whereas the metabolic recovery was incomplete in the UFH group at the end of reperfusion. Complete recovery was reached in this group about 30 minutes after weaning from CPB (Figure 3).

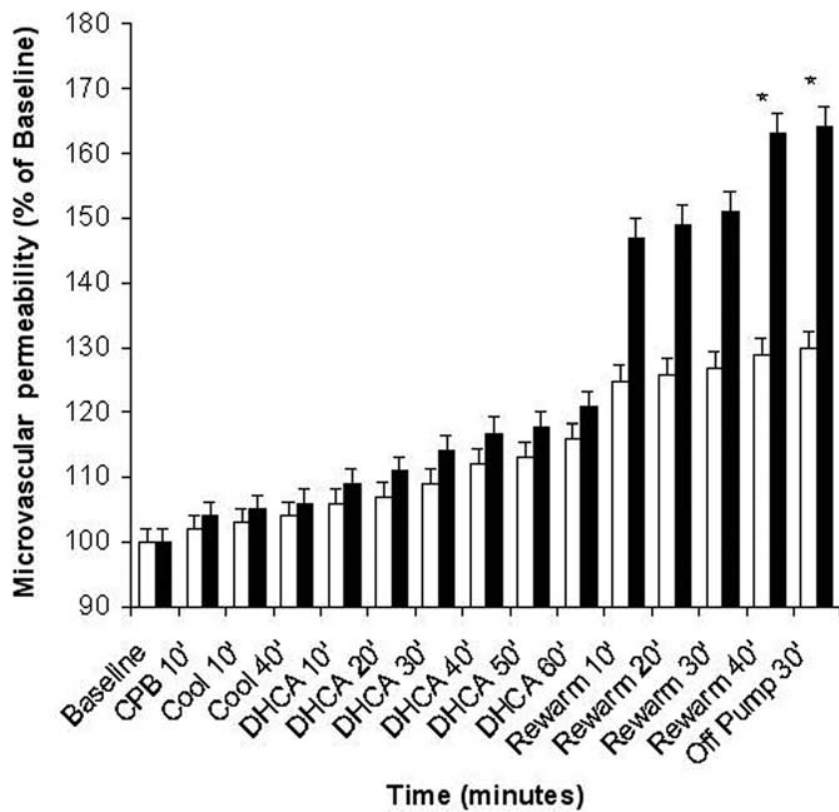

Figure 4. Microvascular permeability was increased in the UFH group during rewarming. This increase has reached statistical significance at 40 minutes of rewarming $(P<.05)$. Filled columns, UFH group; open columns, eptifibatide group. ${ }^{*} P<.05$.
Microvascular Permeability

During cooling and DHCA, there was a slight increase in microvascular permeability in both groups, although this was without statistical significance between the study and control groups. By the end of rewarming, the microvascular permeability had increased significantly in the UFH group compared with that in the eptifibatide group (at 40 minutes of rewarming and 30 minutes after weaning from $\mathrm{CPB} ; P<$ .05 , respectively), indicating a more severe endothelial lining alteration in this group (Figure 4).

\section{Histopathologic Analysis}

Morphologic investigation of hippocampal tissue that was processed for light and electron microscopic analysis by embedding in epoxy resin reveals marked differences in the untreated (UFH) group versus the eptifibatide-treated group. Ultrastructural investigation of the hippocampus confirms and extends findings concerning the superficial cerebrocortical tissue alterations in the UFH group compared with findings in those treated with eptifibatide. Severe damage appeared in the UFH group. Most prominent is the perivascular edema formation and disintegration of the endothelial lining from the surrounding interstitium. In the eptifibatide group these ultrastructural tissue alterations are markedly reduced (Figure 5).

We demonstrate in this experimental study that platelet glycoprotein IIb/IIIa receptor blockade with eptifibatide in the setting of DHCA-reperfusion on CPB during cardiac surgery significantly improves cerebral capillary reperfusion and significantly reduces vascular permeability. The significantly higher FCD values during reperfusion in the eptifibatide group compared with those in the UFH group could likewise be shown. Damage to the endothelial cells on $\mathrm{CPB}$, especially during circulatory arrest, rapidly induces a 

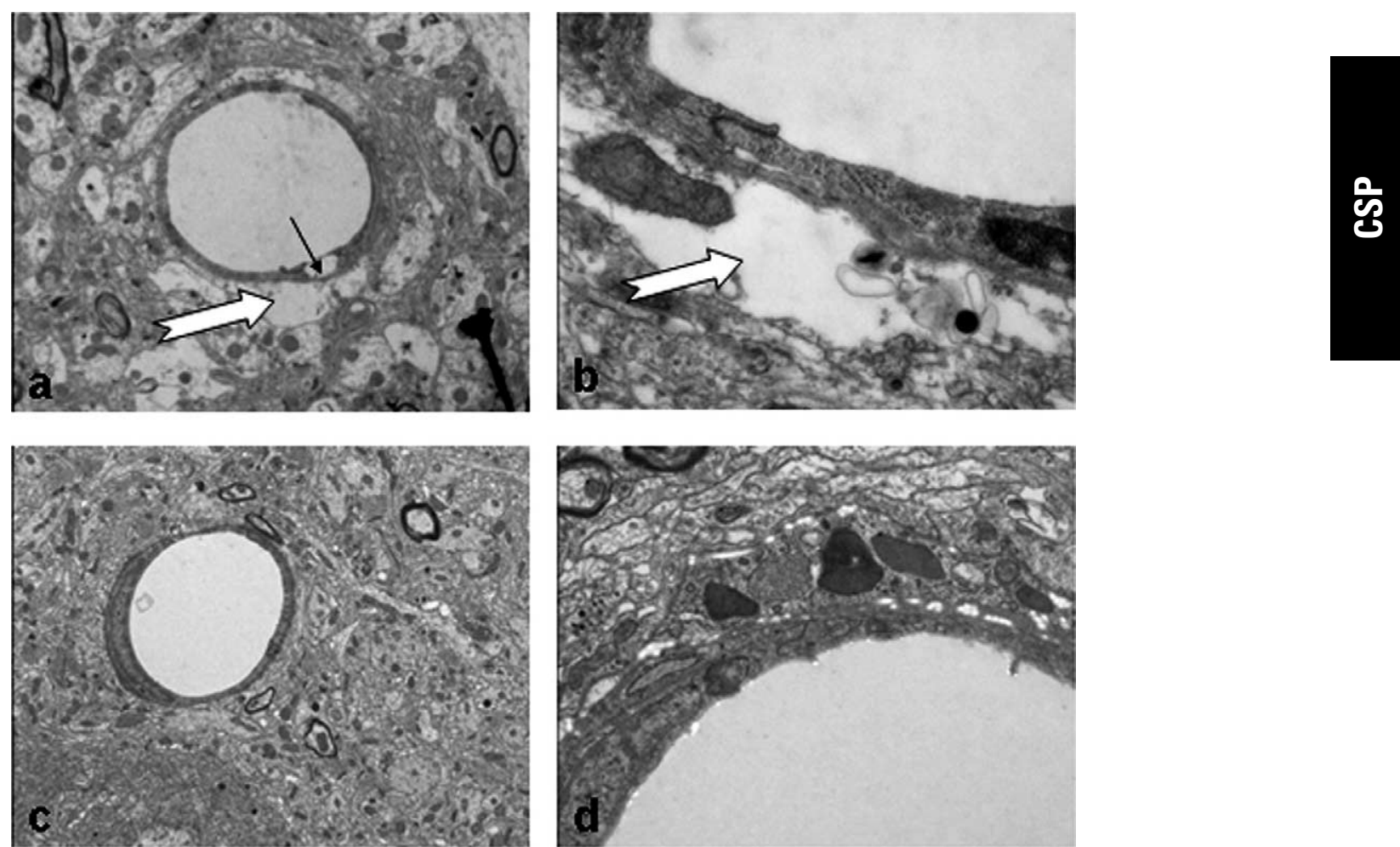

Figure 5. Electron microscopy: ultrastructural investigations of hippocampal tissue (blood vessels) in the UFH group ( $a$ and $b$ ) and the eptifibatide group ( $c$ and d). a, Endothelial degeneration (vacuoles) and perivascular edema are visible. (Original magnification $4400 \times$.) b, At a higher magnification, the perivascular edema is clearly visible. (Original magnification $12,000 \times$.) c, There is mild perivascular edema in eptifibatide-treated animals. (Original magnification $4400 \times$.) d, Only weak perivascular alterations appear in this group. (Original magnification $12,000 \times$.) Black arrow, Vacuole formation; white arrow, perivascular edema formation.

response by the attachment of platelets to the site of the injury coupled to their activation. The initial adhesion and activation occur in a time scale of milliseconds to seconds and require the concerted interaction of matrix proteins with platelet receptors. The main platelet counterparts include collagen receptors, integrin $\alpha 2 \beta 1$, glycoprotein VI, von Willebrand factor receptor, glycoprotein $\mathrm{Ib} \alpha$, and integrin $\alpha \mathrm{IIb} \beta 3$ (glycoprotein IIb-IIIa). ${ }^{12}$ The adherent and activated platelets become the substrate for the second phase of platelet plug formation, which develops in a time frame of minutes and involves the recruitment and activation of additional circulating platelets into the growing thrombus. This phase of homotypic aggregation depends on the formation of interplatelet bonds that involve primarily glycoprotein $\mathrm{Ib} \alpha$ in the glycoprotein Ib-IX-V complex and activated $\alpha \mathrm{IIb} \beta 3$ as receptors on the platelet surface and plasma von Willebrand factor and fibrinogen as the ligands that link platelets to one another. ${ }^{8}$ Platelet inhibition by $\alpha \mathrm{IIb} \beta 3$ (glycoprotein IIb/IIIa) blockade significantly reduces platelet aggregation and activation. This leads to the subsequent significantly higher FCD values in the eptifibatide group compared with those in the UFH group (Figure 1). The preservation of FCD in the eptifibatide group contributes to the more even and homogeneous brain reperfusion (higher RBCV). The no-reflow phenomenon becomes negligible. Cerebral tissue oxygenation was significantly higher in the eptifibatide group during DHCA and early reperfusion. There are several lines of evidence that platelet-endothelium interactions contribute to endothelial dysfunction and impaired nitric oxide bioactivity. ${ }^{13,14}$ Platelet glycoprotein IIb/ IIIa blockade contributes to enhanced nitric oxide bioactivity, which maintains the vasomotor activity. ${ }^{10,15}$ This, associated with higher FCD values in the eptifibatide group, results in enhanced capillary perfusion (higher RBCV), which in turn explains the higher tissue oxygenation in this group. The intravital microscopy also shows higher microvascular permeability in the UFH group relative to the eptifibatide group, which reaches statistical significance at the end of rewarming. This is consistent with the improved histologic findings in the eptifibatide group compared with those in the UFH group. These results support the concept that there is improved structural preservation of the inner 
lining of blood vessels formed by endothelial cells when eptifibatide is used. Platelets have been shown to contain vascular endothelial growth factor (VEGF), which is released during clotting. ${ }^{16}$ Previous studies showed that there is concerted activation between integrins, such as integrin $\alpha \mathrm{v} \beta 3$ and VEGF2. There is a synergistic interaction between the clustered integrin receptor $\alpha \mathrm{v} \beta 3$ and VEGFR2. This is required for full phosphorylation of VEGFR2, which increases the microvascular permeability. ${ }^{17}$ It might be such a mechanism underlying the beneficial effect of eptifibatide by inhibiting integrin $\alpha \mathrm{IIb} \beta 3$ (glycoprotein IIb/IIIa), which binds to multiple substrates, and consequent downregulation of VEGFR2. Further investigations to clarify this mechanism need to be carried out. One of the limitations of this intravital microscopy study of cerebral microcirculation is the fact that only superficial cerebrocortical vessels can be assessed. Subcortical microvessels cannot be studied with this direct method in a nondisruptive manner. ${ }^{11}$ However, hypoperfusion of deeper, selectively vulnerable brain regions, such as the hippocampus, caudate nucleus, and neurons, is most likely important in the pathogenesis of neurologic injury after DHCA. For that reason, we performed histologic investigations (electron microscopy) of hippocampal tissue. These investigations demonstrated that there was a significant increase in histologic evidence of brain injury in the UFH group. The markedly higher extent of microvascular alterations after DHCA-reperfusion on CPB in the UFH group versus the eptifibatide group highlights the beneficial effects of eptifibatide on homotypic and heterotypic platelet aggregation and receptor-modulated vasopermeability and vasomotor activity. These data raise the possibility that the clinical use of eptifibatide in the setting of DHCA during cardiac surgery might have beneficial effects on the neurologic outlook for these patients. There was no difficulty with bleeding during the entire study. Eptifibatide had a protective effect on platelets. A significant preservation of the total platelet count at the end of the experiment was documented in the study group (Table 1). This might dissipate any reluctance for cardiovascular surgeons to use a platelet inhibitor of this potency. The use of eptifibatide in a survival animal model might show the long-term neurologic, cognitive, and behavioral benefits in the setting of DHCA-reperfusion.

We acknowledge the substantive contributions of Hart Lidov at Children's Hospital, Boston. We thank Richard Jonas, MD, for his constructive comments. We further acknowledge the great help of Silke Coburger at the Department of Biostatistics, University of Cologne.

\section{References}

1. Liu S, Connor J, Peterson S, Shuttleworth CW, Liu KJ. Direct visualization of trapped erythrocytes in rat brain after focal ischemia and reperfusion. J Cereb Blood Flow Metab. 2002;22:1222-30.
2. Del Zoppo GJ, Mabuchi T. Cerebral microvessel responses to focal ischemia. J Cereb Blood Flow Metab. 2003;23:879-94.

3. Gunther A, Manaenko A, Franke H, Wagner A, Schneider D, Berrouschot J, et al. Hyperbaric and normobaric reoxygenation of hypoxic rat brain slices-impact on purine nucleotides and cell viability. $\mathrm{Neu}$ rochem Int. 2004;45:1125-32.

4. Ames MN, Senning A. Studies in oxygen consumption during extracorporeal circulation with a pump-oxygenator. Ann Surg. 1958;148: 59-65.

5. Norwood WI, Norwood CR, Castaneda AR. Cerebral anoxia: effect of deep hypothermia and pH. Surgery. 1979;86:203-9.

6. Kourembanas S, Marsden PA, McQuillan LP, Faller DV. hypoxia induces endothelin gene expression and secretion in cultured human endothelium. J Clin Invest. 1991;88:1054-7.

7. Gerler JP, Ocasio VH. Endothelin production by hypoxic human endothelium. J Vasc Surg. 1993;18:178-82; discussion 182-4.

8. Ruggeri ZM. Platelet and von Willebrand factor interactions at the vessel wall. Haemostaseologie. 2004;24:1-11.

9. Cunningham JN, Adams PX, Knopp EA, Baumann FG, Snively SL, Gross RI, et al. Preservation of ATP, ultrastructure and ventricular function following aortic cross-clamping and reperfusion: clinical use of blood potassium cardioplegia. J Thorac Cardiovasc Surg. 1979;78:708-20.

10. Heitzer T, Ollmann I, Koeke K, Meinertz T, Munzel T. Platelet glycoprotein IIb/IIIa receptor blockade improves vascular nitric oxide bioavailability in patients with coronary artery disease. Circulation. 2003;108:536-41.

11. Duebener LF, Hagino I, Sakamoto T, Ben Mime L, Stamm C, Zurakowski D, et al. Effects of $\mathrm{pH}$ management during deep hypothermic bypass on cerebral microcirculation: alpha-stat versus $\mathrm{pH}$-stat. Circulation. 2002;106(suppl I):I103-8.

12. Ruggeri ZM. Platelets in atherothrombosis. Nat Med. 2002;8:1227-34.

13. Cohen RA, Shepherd JT, Vanhoutte PM. Inhibitory role of the endothelium in the response of isolated coronary arteries to platelets. Science. 1983;221:273-4.

14. Houston DS, Shepherd JT, Vanhoutte PM. Aggregating human platelets cause direct contraction and endothelium-dependent relaxation of isolated canine coronary arteries: role of serotonin, thromboxane A2, and adenine nucleotides. J Clin Invest. 1986;78:539-44.

15. Pu Q, Wiel E, Corseaux D, Bordet R, Azrin MA, Ezekowitz MD, et al. Beneficial effect of glycoprotein IIb/IIIa inhibitor (AZ-1) on endothelium in Escherichia coli endotoxin-induced shock. Crit Care Med. 2001;29:1181-8.

16. Banks RE, Forbes MA, Kinsey SE, Stanley A, Ingham E, Walters C, et al. Release of the angiogenic cytokine vascular endothelial growth factor (VEGF) from platelets: significance for VEGF measurements and cancer biology. Br J Cancer. 1998;77:956-64.

17. Masson-Gadais B, Houle F, Laferriere J, Huot J. Integrin alphavbeta3, requirement for VEGFR2-mediated activation of SAPK2/ p38 and for Hsp90-dependent phosphorylation of focal adhesion kinase in endothelial cells activated by VEGF. Cell Stress Chaperones. 2003;8:37-52.

\section{Discussion}

Dr James W. Frederiksen (Chicago, Illinois). This is nice work. I would like to know whether you used eptifibatide in the setting of bypass at normothermia without circulatory arrest?

Dr Ben Mime. Actually, we have a study going in which we are using eptifibatide at normothermia without circulatory arrest.

Dr Frederiksen. I think the use of eptifibatide in this setting of bypass at normothermia could improve the microcirculation.

Dr Ben Mime. It might be 